\title{
Ciguatera fish poisoning after Caribbean travel
}

\author{
Courtney A. Thompson MD MSc, Farah Jazuli MSc, Linda R. Taggart MD MPH, Andrea K. Boggild MSc MD
}

Cite as: CMAJ 2017 January 9;189:E19-21. doi: 10.1503/cmaj.151207

\section{Case 1}

A previously healthy 39-year-old Canadian-born man travelled to Havana, Cuba, for one week on business. He stayed in a local home and ate foods prepared by the family. The night before his departure home, he ate two portions of a large fish identified by the host as a dog snapper (Figure 1). Two days after his return, he noted prominent symptoms of temperature inversion (i.e., reversal of hot and cold sensations) in his upper and lower extremities: his hands felt subjectively hot and "burning" when he washed them in cold water, he was unable to discern hot from cold face cloths, and he experienced "burning hot" feet while walking barefoot on cold, tiled floors. The symptoms of temperature reversal did not occur when he ate or drank and were not prominent when he showered. $\mathrm{He}$ also described a tingling sensation around his mouth and nose, as well as painful joints, particularly his knees and elbows. He had no gastrointestinal symptoms, rash or palpitations.

On examination, the patient was well and alert and oriented to person, time and place. He was afebrile and had a regular pulse. There was no facial asymmetry, and sensation to light touch was normal throughout. He had full power in his upper and lower extremities, and his gait was normal. There was no rash, and his joints were not red or swollen. A clinical diagnosis of ciguatera was made, and the patient was advised to avoid consuming fish, nuts, caffeine and alcohol for at least six months.

His symptoms continued intermittently, with noted exacerbations following heavy exercise and alcohol consumption. Exacerbations occurred on three occasions over the subsequent month

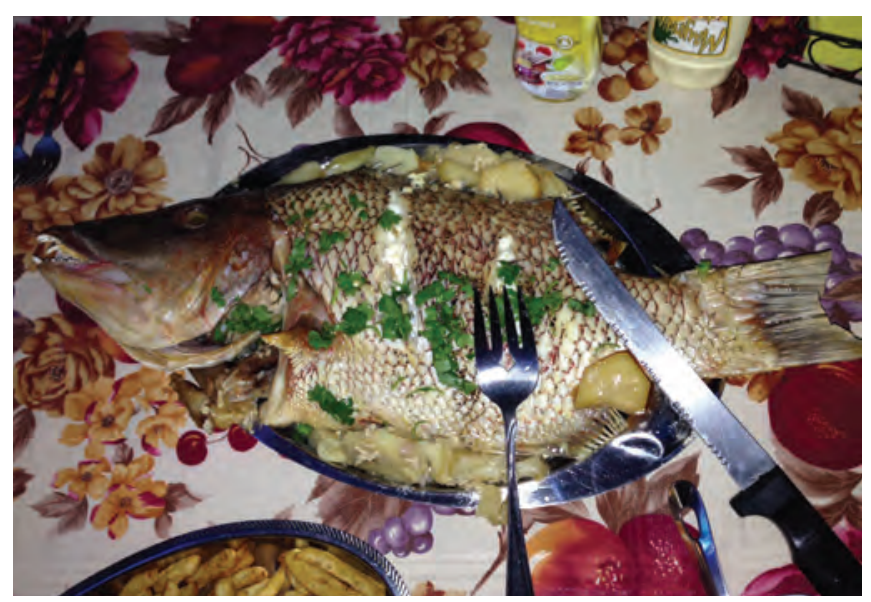

Figure 1: Dog snapper eaten by a 39-year-old man while in Cuba (case 1).

\section{KEY POINTS}

- Ciguatera is a marine toxicity that may occur following ingestion of large reef fish such as snapper, barracuda, grouper and eel.

- Sensation of hot-cold temperature reversal to liquids and solid objects is the pathognomonic sign of ciguatera, but it is present in only $50 \%$ of those affected.

- Toxins are not destroyed by cooking or freezing; therefore, improper food preparation, handling and storage are not implicated in the pathogenesis of ciguatera.

- Diagnosis is clinical and rests on compatible epidemiology and the presence of characteristic signs and symptoms, such as sensation of hot-cold temperature reversal.

- Management is supportive.

and were characterized by worsening joint pain, tingling and paresthesia of the face and extremities, and insomnia. By six weeks after onset, the symptoms had resolved entirely, and the patient returned to his normal diet without restrictions. He remained well at six weeks, and three and six months after symptom resolution.

\section{Cases 2 and 3}

A husband and his wife, both Guyanese-born and 68 years old, travelled to the Dominican Republic for one week to visit family and friends. While there, they purchased and ate local fish that was cooked and sold on a rural beach close to Santo Domingo. About four hours later, they and their friends began to feel unwell with nausea, vomiting and diarrhea. About two days later, the husband and wife experienced severe generalized pruritus. Two days after that, both began to have unusual sensations when drinking cold liquids: the husband had an odd sensation on his tongue, and the wife had symptoms of temperature reversal. She reported that her hands felt burning hot while rinsing them in cold water, and her husband experienced perioral numbness, weakness of his hands and a cold sensation of his left foot. Both were evaluated in Canada in local emergency departments before referral to a tropical disease unit at an academic tertiary care centre. The husband underwent a full stroke work-up for transient numbness of his left arm and leg, the results of which were negative. Hydroxyzine was prescribed to both patients for the pruritus, which led to mild improvement.

In follow-up in the tropical disease unit, about one month after symptom onset, both patients appeared well, were afebrile and had a regular pulse. Findings on cranial nerve examination were 
normal. The patients had full power in their upper and lower extremities and had a normal gait. The husband had mild reduction to light touch sensation of the left foot. Neither had a rash, or red or swollen joints. A clinical diagnosis of ciguatera was made, and the patients were advised to avoid consuming fish, nuts, caffeine and alcohol for at least six months.

Adherence to caffeine avoidance proved challenging, but otherwise both patients were able to reduce their consumption of dietary triggers, and neither had exacerbation of symptoms. By two months after the onset of symptoms, the pruritus had improved in both, and the wife's temperature reversal and husband's perioral tingling were mild. By four months, their symptoms had almost completely resolved, and both began to reintroduce fish to their diet.

\section{Discussion}

Ciguatera is a food-borne illness that occurs in inhabitants of, and visitors to, islands surrounded by coral reefs. The incidence among Canadians returning from high-risk regions is unclear, because it is not a reportable illness. Ciguatera is the most prevalent type of fish poisoning and has the lowest mortality. A total of 51 cases were reported to the global GeoSentinel surveillance network over a five-year period, in contrast to 7 cases of scombroid fish poisoning and neurotoxic or paralytic shellfish poisoning in the same period. ${ }^{1}$ However, it is likely that large numbers of cases go undiagnosed or unreported.

\section{Pathogenesis}

Ciguatoxins have no odor and are not destroyed by cooking or freezing. They originate from plankton species in the genus Gambierdiscus found on coral reefs. ${ }^{2}$ The toxins are transmitted to humans via ingestion of large reef fish such as grouper, snapper, barracuda and eel. ${ }^{3}$ Unlike other food-borne intoxications (e.g., botulism), improper handling, storage and preparation of fish are not implicated in the pathogenesis of ciguatera. Outbreaks commonly occur in restaurant or banquet settings because many people may ingest large portions of the affected fish.

Although ciguatoxins are predominately concentrated in the Caribbean, they are found in the Pacific and Indian Oceans as well (Figure 2).

\section{Clinical manifestations}

Temperature reversal is the pathognomonic symptom of ciguatera, but it occurs in only $50 \%$ of cases; thus, its absence does not preclude the diagnosis. ${ }^{4}$ Temperature reversal is the sensation that cold objects feel hot or induce dysesthesia and vice versa. Other symptoms of ciguatera include cutaneous, gastroenterologic, neurologic and cardiac manifestations, with symptom severity directly proportional to the amount of ingested toxin (Box 1). Hospital admission is sometimes necessary because of severe bradycardia with hypotension, which occurs in a minority of reported cases. Symptoms and duration of illness may vary by geographic region of acquisition. Gastrointestinal symptoms predominate from ciguatera acquired in the Caribbean, whereas neurologic and cardiovascular symptoms are more prominent in ciguatera from the Pacific and Indian Oceans. ${ }^{5,6}$

\section{Differential diagnosis}

The diagnosis of ciguatera fish poisoning can be challenging in the absence of the pathognomonic finding of temperature reversal and history of the type of fish eaten. Poisonings from other marine toxins from different fish and seafood species may present with similar clinical features, but they usually have a more rapid onset than ciguatera. Paralytic shellfish poisoning, neurotoxic shellfish poisoning, scombroid fish poisoning and puffer fish poisoning typically present within minutes to a few hours after ingestion. Ciguatera has

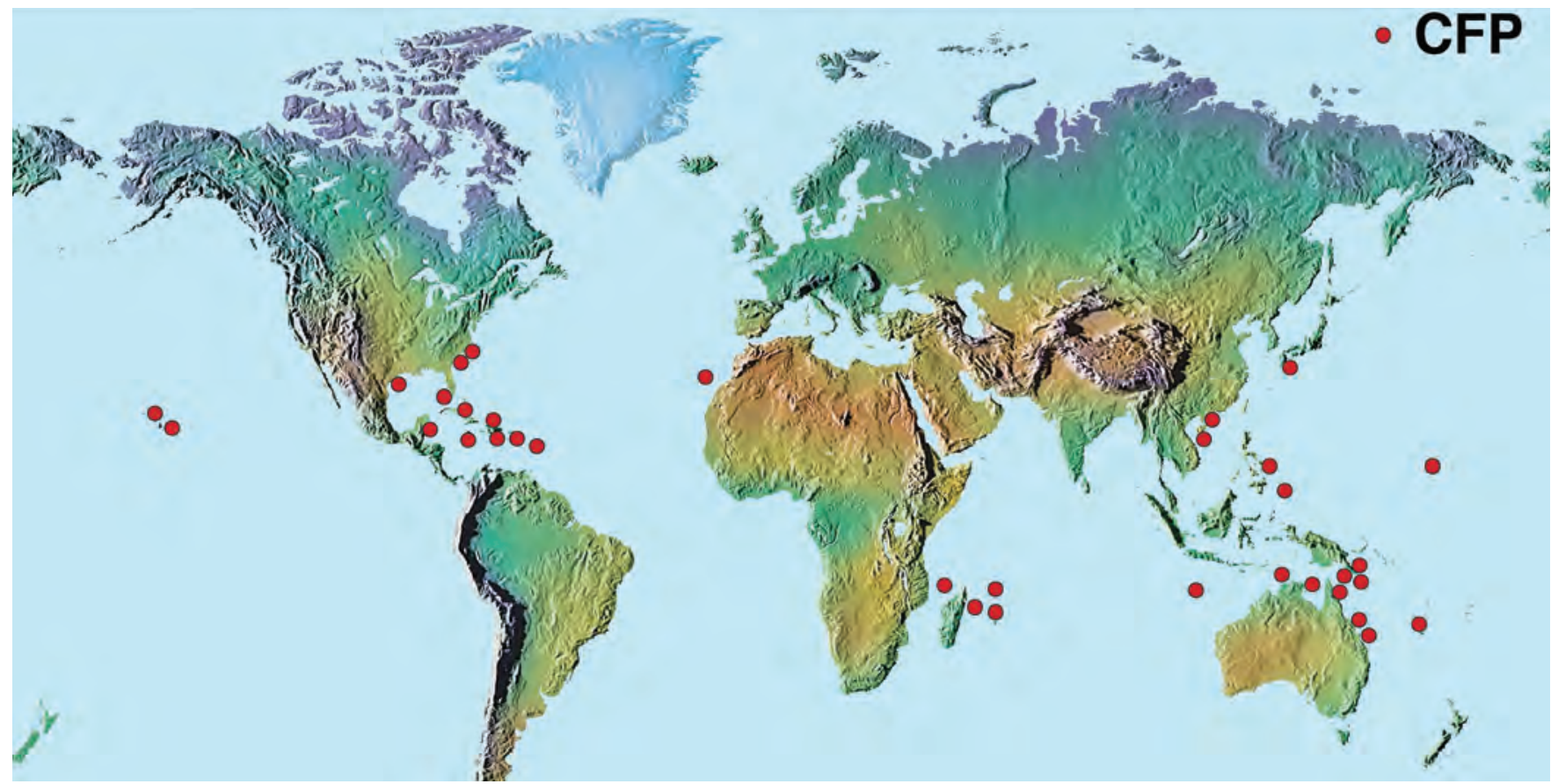

Figure 2: Global distribution of ciguatera fish poisoning (CFP). Reproduced with permission from the US National Office for Harmful Algal Blooms, Woods Hole Oceanographic Institution, Woods Hole, Mass. 


\section{Box 1: Clinical characteristics of ciguatera ${ }^{3}$}

\section{Symptoms}

Time to resolution

Neurologic: Paresthesia (numbness and tingling) in the extremities and around or near the mouth, sensation of temperature reversal (hot-cold inversion), ${ }^{\star}$ ataxia, headache

Gastrointestinal: Nausea, vomiting, diarrhea, $\quad 1-4 \mathrm{~d}$ (typically $\leq 24 \mathrm{~h}$ ) abdominal cramping

Cardiovascular: Bradycardia, heart block, $1-3 d$ hypotension

Other: Myalgia, generalized pruritus, arthralgia, fatigue, dizziness, dysuria

*The sensation that cold objects feel hot or result in dysesthesia (abnormal sensation) or vice versa. Although this phenomenon is not present in all patients, it is considered pathognomonic of ciguatera.

a more delayed onset, from several to 12 hours on average, and in some cases 24-48 hours after ingestion (Appendix 1, available at www.cmaj.ca/lookup/suppl/doi:10.1503/cmaj.151207/-/DC1).

If present, the sensation of temperature reversal distinguishes ciguatera from other similar conditions. Symptoms are otherwise nonspecific and have considerable overlap with other diagnoses. Illnesses caused by nonmarine toxins, such as organophosphate poisoning or botulism, share gastrointestinal and neurologic features with ciguatera. Early presentations of multiple sclerosis with predominant sensory symptoms may be initially difficult to distinguish from ciguatera before the onset of additional symptoms. In patients whose symptoms persist for months, a misdiagnosis of chronic fatigue syndrome may occur. A thorough exposure history is helpful in narrowing the differential diagnosis.

\section{Diagnosis}

The diagnosis of ciguatera is clinical. Sophisticated investigations of paresthesia, such as nerve conduction studies and magnetic resonance imaging of the brain, are unnecessary and not helpful. ${ }^{7}$ Diagnosis relies on the identification of symptoms typical of ciguatera combined with appropriate exposure or travel history. Laboratory diagnosis is typically unavailable, and clinical suspicion is sufficient. Definitive diagnosis is usually unnecessary, because treatment options for marine intoxications are largely supportive. Laboratory detection of the toxin in remaining fish or shellfish may be undertaken in outbreak settings when such samples are available for testing.

\section{Management}

Management of the ambulatory patient with ciguatera rests on avoidance of potential exacerbating triggers. Although large rigorous studies are lacking, reports of consumption of caffeine, peanuts, ${ }^{8}$ seafood ${ }^{8,9}$ and alcohol ${ }^{8,9}$ have all been associated with worsening of symptoms, as has exercise. ${ }^{3}$ Although previous studies have suggested mannitol infusion as a treatment for neurologic symptoms of ciguatera, a randomized controlled trial found no difference in outcome between patients given mannitol and controls given normal saline. ${ }^{10}$

Patient-level prevention includes education on the avoidance of consuming large tropical reef fish. Beyond complete avoidance of implicated marine species, risk can be reduced by eating smaller fish $(<2 \mathrm{~kg})$ and by avoiding parts of fish that tend to accumulate the toxin, such as the head, roe, skin and visceral organs. Large-scale preventive interventions, such as greater global regulation of marine trade and diligence surrounding environmental monitoring, require broad international coordination and engagement.

\section{Conclusion}

Ciguatera fish poisoning, among other marine poisonings, is underrecognized in Canada, which can lead to potentially unnecessary investigations and morbidity in the patient. Early recognition of the identifying clinical features of ciguatera can result in improved patient care and more efficient use of resources.

\section{References}

1. Leder K, Torresi J, Libman MD, et al. GeoSentinel surveillance of illness in returned travelers, 2007-2011. Ann Intern Med 2013;158:456-68.

2. Litaker RW, Vandersea MW, Faust MA, et al. Global distribution of ciguatera causing dinoflagellates in the genus Gambierdiscus. Toxicon 2010;56:711-30.

3. Dickey RW, Plakas SM. Ciguatera: a public health perspective. Toxicon 2010;56: 123-36.

4. Friedman MA, Fleming LE, Fernandez M, et al. Ciguatera fish poisoning: treatment, prevention and management. Mar Drugs 2008;6:456-79.

5. Pottier I, Vernoux JP, Lewis RJ. Ciguatera fish poisoning in the Caribbean islands and western Atlantic. Rev Environ Contam Toxicol 2001;168:99-141.

6. Skinner MP, Brewer TD, Johnstone R, et al. Ciguatera fish poisoning in the Pacific Islands (1998 to 2008). PLoS Negl Trop Dis 2011;5:e1416.

7. Isbister GK, Kiernan MC. Neurotoxic marine poisoning. Lancet Neurol 2005;4: 219-28.

8. Liu Z, Wei H, Lin Z, et al. Clinical analysis of a collective ciguatoxin poisoning accident in a banquet [Chinese]. China Trop Med 2006;9:1689-91.

9. De Haro L, Hayek-Lanthois M, Joossen F, et al. Mass ciguatera poisoning after eating barracuda in Mexico: prognostic and therapeutic implications. Med Trop (Mars) 1997;57:55-8.

10. Schnorf H, Taurarii M, Cundy T. Ciguatera fish poisoning: a double-blind randomized trial of mannitol therapy. Neurology 2002;58:873-80.

\section{Competing interests: None declared.}

This article has been peer reviewed.

The authors have obtained patient consent.

Affiliations: London School of Hygiene \& Tropical Medicine (Thompson), London, UK; Faculty of Medicine (Jazuli), University of Toronto; Department of Medicine (Taggart, Boggild), University of Toronto; Division of Infectious Diseases (Taggart), St. Michael's Hospital, Toronto, Ont.; Tropical Disease Unit, Division of Infectious Diseases, Department of Medicine (Boggild), Toronto General Hospital, University Health Network; Public Health Ontario Laboratories (Boggild), Toronto, Ont.
Contributors: Andrea Boggild and Courtney Thompson conceived and drafted the manuscript. All of the authors contributed to the literature review, revised the manuscript critically for important intellectual content, approved the final version to be published and agreed to act as guarantors of the work.

Acknowledgement: The authors gratefully acknowledge Rachel Lau and Jennifer Ma for their assistance with translating reference 8.

Correspondence to: Andrea Boggild, andrea.boggild@utoronto.ca 